\title{
A Successful Senior Seminar: Unsolved Problems in Number Theory
}

\author{
Robert Styer \\ Department of Mathematics and Statistics \\ 800 Lancaster Avenue \\ Villanova University \\ Villanova, PA 19085, USA \\ robert.styer@villanova.edu
}




\title{
A Successful Senior Seminar: Unsolved Problems in Number Theory
}

\begin{abstract}
The Unsolved Problems in Number Theory book by Richard Guy provides nice problems suitable for a typical math major. We give examples of problems that have worked well in our senior seminar course and some nice results that senior math majors can obtain. We conclude with a brief overview of how we implement our seminar.
\end{abstract}

Keywords: number theory, happy numbers, Gaussian primes, multiplicative persistence

\section{INTRODUCTION}

Many math departments at schools similar to Villanova University require a senior seminar (capstone course) with goals such as these:

- Experience doing research and reading original literature,

- Learn to use MathSciNet and other library tools,

- Experience giving oral presentations,

- Learn mathematical writing, in particular, how to write theorems/proofs,

- tie together ideas from previous courses in a new context.

The author's most successful implementation of the seminar uses Richard Guy's Unsolved Problems in Number Theory (UPINT) [1]. Guy's book contains about 170 unsolved problems with extensive lists of references, and the problems are easily stated and understood by our students. Of course, a handful of our best math majors choose topics like computing small Galois groups, Ramsey theory, or Hilbert's Twelfth 
Problem. But most of our students do not go to graduate school in mathematics, and Guy's book contains several problems that will engage them for a full semester. Students tend to choose problems based on the "cuteness factor" rather than the content of the problem; students choose happy numbers, lucky numbers, Ruth-Aaron numbers, magic squares, good primes, multiplicative persistence of a number. Students also love problems cast as games or puzzles such as Mousetrap, the $n$-Queens problem, Epstein's Put or Take a Square Game, or No Three in a Line. Students investigate the problems numerically, then research the literature on their problems. Recreational math topics allow typical math majors a lot of room to explore new territory and a smaller and more accessible research literature; cute and obscure problems are good!

\section{Example: happy numbers}

Iterative digit functions make nice research problems. For instance, many students choose to investigate "happy numbers" (UPINT E34). Consider the digit function $S(N)=\sum_{j=0}^{r} a_{j}^{2}$ where $N=\sum_{j=0}^{r} a_{j} 10^{j}$ and each digit satisfies $0 \leq a_{j} \leq 9$. For instance, $S(44492)=4^{2}+4^{2}+$ $4^{2}+9^{2}+2^{2}=133$. Iterating this, $S(133)=19, S(19)=82, S(82)=70$, $S(70)=49, S(49)=97, S(97)=130, S(130)=10, S(10)=1$ and from here on we continue getting 1 . When a number iterates to 1 we call it 'happy' so 44492 is a happy number. The iterates of 44493 are $44493 \rightarrow 138 \rightarrow 74 \rightarrow 65 \rightarrow 61 \rightarrow 37 \rightarrow 58 \rightarrow 89 \rightarrow 145 \rightarrow 42 \rightarrow 20 \rightarrow$ $4 \rightarrow 16 \rightarrow 37 \rightarrow 58$ where we now see a cycle of length eight. Such numbers are said to be 'unhappy' though a cuter name would be '4-lorn' since one can show all numbers are either happy or go to this cycle with smallest number 4 .

Questions students can easily investigate are:

- are there any other cycles? (no)

- what is the density of happy numbers? (unsolved; seems to be about 1 out of 7 )

- what happens in other bases? (lots of research potential) 
- what happens if we modify $S(N)$, e.g., cubes or triangular numbers replacing squares?

- what is the longest string of consecutive happy numbers? (arbitrarily long)

- what is the first string of six happy numbers?

A student project in 2007 inspired the author to consider the last question. We first note that if happy numbers were randomly distributed with a density of $1 / 7$, then we might expect a string of five consecutive happy numbers around $N=7^{5}=16807$ and one can calculate that the first string of five consecutive happy numbers is 44488, 44489, 44490, 44491, and 44492. So one might expect that the first string of six consecutive happy numbers might be around $7^{6}=117649$. It is easy for students to write a program to search for the first string of six consecutive happy numbers but it shows the futility of blind searching: the least example begins with $N=7899999999999959999999996$. The author found a computational method to locate the least positive integer that begins a length $k$ sequence of consecutive happy numbers for $k=6$, $7,8,9,10,11,12$, and 13 . His method, however, fails for $k=14$ since it would require roughly $10^{15}$ calculations.

In 2012, a student insisted that he wanted to find the least number starting a sequence of 14 consecutive numbers. About midsemester he had an idea; when calculating $S(N)$ the order of the digits of $N$ is not relevant. He applied this obvious fact in a nonobvious way to cut the number of computations from $10^{15}$ to less than $10^{8}$, a very computable number. His methods also found the least example for 15 consecutive happy numbers. His paper was accepted for publication by Involve: $A$ journal of mathematics [2].

The published research on happy numbers deals with theoretical questions such as proving that arbitrarily long sequences of generalized happy numbers are possible for arbitrary bases and for many classes of iterative digit functions $S$. Mathematicians tend to ignore specific problems such as the least example of 14 consecutive happy numbers in base 10. Specific problems encourage students to compute, and often lead 
them to discover new results. The results are perhaps not earth-shaking but students derive a great deal of satisfaction from knowing that they have discovered a result unknown by anyone before them.

\section{Second example: multiplicative persistence}

Another iterative digit problem is multiplicative persistence (UPINT F25). One multiplies the digits of a number until one reaches a single digit. Consider the digit function $T(N)=\prod_{j=0}^{r} a_{j}$ where $N=$ $\sum_{j=0}^{r} a_{j} 10^{j}$ and each digit satisfies $0 \leq a_{j} \leq 9$. For instance, $T(6788)=$ $6 \cdot 7 \cdot 8 \cdot 8=2688$. Iterating this function, $T(2688)=768, T(768)=336$, $T(336)=108$, and $T(108)=0$. We say that 6788 has persistence 5 since it took five iterations to reach a one digit number (a fixed point).

Some questions suitable for student investigation:

- Is the persistence bounded?

- If it is bounded, what is the maximum persistence?

- Is persistence well defined (that is, does one necessarily iterate to a single digit)?

- What is the persistence in other bases?

- If we disallow zero digits, what is the maximum persistence? (a question of Erdös)

- If one modifies the function $T$ (for instance, multiply the square or cube of each digit) does one eventually iterate to a single digit?

- Which numbers iterate to a particular digit such as 6 ?

In 1973 Sloane conjectured that the maximum persistence is 11 . He calculated that no number under $10^{50}$ has persistence greater than 11 ; one of the seminar students showed no number under $10^{1500}$ has persistence exceeding 11. Conjecturally (see [3]) the maximal persistence in bases 2 through 12 are $1,3,3,6,5,8,8,6,7,11,13,7$; most of these conjectures do not seem to be in the literature. This problem involves accessible proofs and conjectures, results are amenable to calculation, and as with most recreational math problems, the literature is not difficult to read. 


\section{Third example: Gaussian primes}

Guy's beautiful pictures (UPINT A13) of the Gaussian primes and Eisenstein primes generally capture at least one student's interest in the seminar. In 2012 a physics major and math minor who loved programming fell in love with the Gaussian prime picture. He wrote an efficient program to locate and count and draw pictures of the Gaussian primes in various rectangles. At large distances from the origin he noted a decrease in the density of Gaussian primes. He conjectured what their density should be, based on the arguments for the density of primes, and gathered extensive statistics to support his conjectures.

He also noted various recurring shapes, and eventually focused on Gaussian prime triangles as a rough analogy to twin primes: $\mathbf{\square}$. He gathered statistics on the density of Gaussian prime triangles as a function of distance from the origin, and developed a heuristic argument for the density analogous to the argument for the density of twin primes. His colleagues were most impressed, however, by the pictures he used to illustrate his findings. The next four pictures show the Gaussian primes and triangles at various distances from the origin (Gaussian composites are black, Gaussian primes are white unless the prime is the vertex of a Gaussian prime triangle in which case the square is colored gray). The first is located near the origin, the second is distance $10^{4}$, the third is distance $10^{9}$, and the fourth is distance $10^{18}$. These pictures nicely illustrate the decreasing density of Gaussian primes and the more rapidly decreasing density of Gaussian prime triangles.

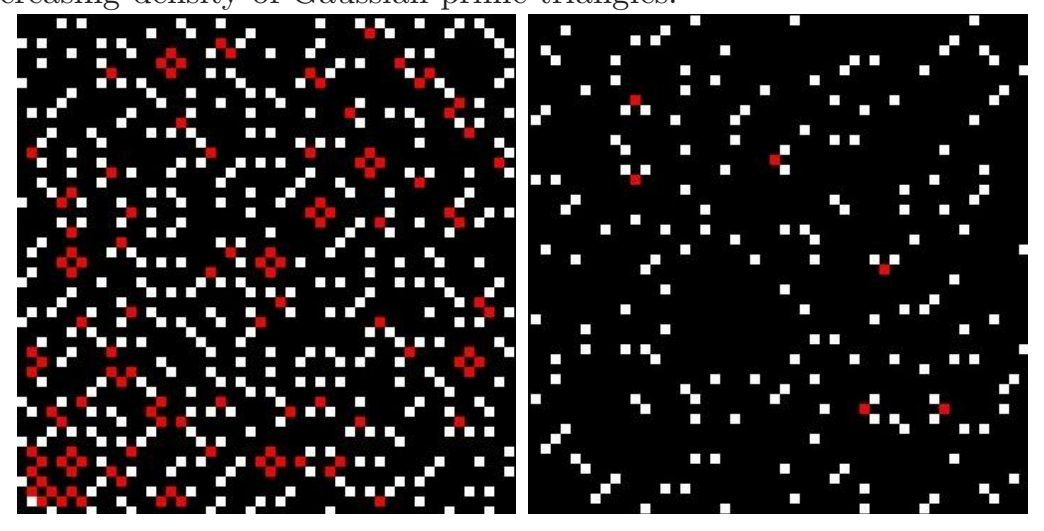



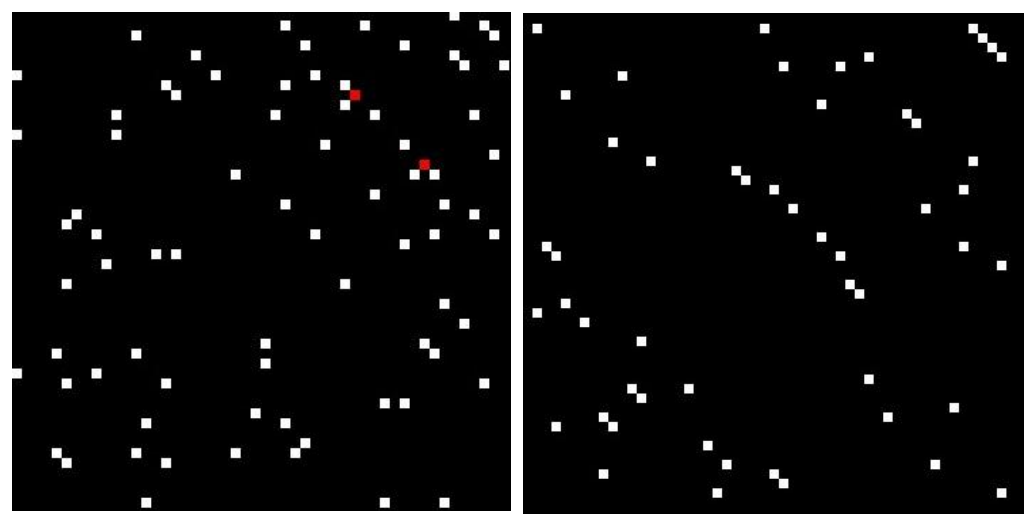

This project indicates what a top student can do; the previous two sections discussed work by students with GPAs in the B range. Because their chosen problem fascinated them, however, they each invested the effort needed to produce a quality final presentation and paper.

\section{Other examples of accessible problems}

In our experience, student engagement with the problem far outweighs the particular problem chosen, since every problem has variants which are amenable to computing, developing conjectures, and proving simple propositions. For instance, another problem that works well with typical math majors is Euler's perfect cuboid problem (UPINT D18). Euler found examples of rectangular boxes all of whose edges and the diagonals of each face have integral length. Can one also find a box with integral main diagonal? This problem opens up many questions whose geometric nature excites students: can the student

- characterize boxes with the three edges and the main diagonal integral?

- find a box with all four diagonals integral though not necessarily the edges?

- find a box with integral sides and main diagonals plus two of the face diagonals?

- characterize integral-sided tetrahedra? 
- determine possible integrality of various subsets of lengths associated with a four dimensional box?

- determine possible integrality of edges and diagonals for various two dimensional figures?

Egyptian fractions perennially attract interest; an Egyptian fraction expansion writes a rational number as a sum of rational numbers all with numerator one and with distinct denominators. For instance, $3 / 5=1 / 2+1 / 10$ and $3 / 7=1 / 3+1 / 11+1 / 231$. The famous ErdösStraus conjecture says that $4 / n$ for all odd integers $n>4$ can always be represented by an Egyptian fraction expansion with three terms. This leads to numerous questions that could spark student exploration:

- Why did Erdös and Straus consider $4 / n$ ? what about $2 / n$ and $3 / n$ ?

- What about $5 / n$ or $6 / n$ ?

- Are there any rational numbers that require more than three terms?

- What is the greedy algorithm and what results can it yield?

- The Egyptians allowed $2 / 3$ as a "unit fraction"; what if we allow it?

- More generally, what if we allow any term of the form $1 / n$ or $2 / n$ ?

Games attract student interest, and one cute though obscure game that math majors enjoy is Epstein's Put or Take a Square Game (UPINT E26). In this game two players alternately remove or add to a pile the largest square value of the pile. For instance, if the pile has 6 stones, one can add or subtract 4 stones. A player wins by taking all the stones. If the pile has 5 stones, the next player can add 4 or subtract 4 . In this case, the following player has either 1 stone or 9 stones, both perfect squares, so in either case the player takes all the stones and wins. In addition to investigating the original problem, students can study variations such as Put or Take a Triangular Number, Put or Take a Prime, Put or Take a Cube, Put or Take a Power of Two. Some variations on the original problem make the analysis much easier and allow the student to discover their own simple conjectures and proofs. 
Another game-related project is the $n$-queens problem (UPINT C18). After the students butt their head against the original problem for a week, one can guide them to try different pieces, or different sizes or shapes of boards, or use combinations of pieces with weights (such as queen worth 9 and rook worth 5) and maximize or minimize the total weight. The student can experiment with one of these variations on small boards, develop their own conjectures, and then can appreciate the clever combinatorial ideas used in the literature to partially solve the original $n$-queens problem.

In summary, many problems invite students to explore computationally, and to adjust the problem and discover the consequences of these changes. Even the simplest problem may have many rich variants that can lead a student to original results.

\section{Seminar Implementation details}

In the past 25 years, the author has taught the seminar a dozen times. The first three weeks introduce some basic ideas in number theory, emphasizing those topics that recall previous course work. For instance, the first day we review Euclid's proof for an infinitude of primes that the students saw in the sophomore Foundations course. The second day we use Maple to investigate the density of primes, concluding with a statement of the Prime Number Theorem. We follow this with an outline of Euler's proof for an infinitude of primes; in addition to providing an excuse to mention the Riemann Hypothesis, this proof allows us to mention geometric series, the Taylor series for the log, the $p$-test, and lots of handwaving where we emphasize that a "real proof" would require the epsilons and deltas they learned in advanced calculus. The next topics are modular arithmetic and Fermat's Little Theorem, providing an excuse to review cyclic groups and subgroups from abstract algebra.

The author's website [4] has the full list of topics introduced in these three weeks. During these three weeks, the students also read all the problems in the UPINT text [1] and a few articles on mathematical 
writing. On the last day of this whirlwind overview of number theory, the students submit a one page writeup identifying the problem they have chosen to investigate.

By the fourth week we begin the talks which occupy the remainder of the semester. The first round features 10 minute talks by each student, introducing his or her problem to the class. At the conclusion of each short talk, we spend a couple minutes giving feedback on how to improve the talk. Generally, the class is Monday-Wednesday-Friday 50 minute periods and the seminar has a limit of fifteen students, so this takes about four 50 minute periods (although sickness sometimes delays a student talk, so the syllabus includes an extra day at the end of each round of talks for makeups).

Then we begin the second round of 20 minute talks, focusing on their personal investigations and calculations related to the problem. The students must set up an office hour with me before this talk, at which time I assist them with Maple coding and encourage them to consider special cases and more specific questions. Most students present their talk with Powerpoint along with occasional whiteboard work, though a few use the whiteboard exclusively while one or two others use Prezi or pdfs (the classroom has a computer projector and docking station). The students in the audience fill out a feedback form developed by the department's most experienced seminar professor, colleague Alice Deanin. After each talk, we briefly review any highlights (mostly just the positive comments) after the talk, then the student speaker receives all the forms, reviews them, and returns them to me the next class. I use the forms to help grade the student talks and as an attendance roster.

After midterm break, the students turn in their first draft of their paper, a 5-10 page writeup of the patterns they have discovered so far. We also begin the third round of 20 minute talks, which focus on the students' library research. A week or two before midterms, the math research librarian spends a class period to introduce MathSciNet, MathWorld, Scopus, and ERIC. The students also have the references listed in UPINT [1] and in Wikipedia. (Some obscure topics, such as multiplicative persistence, have few or no references in Guy and appear in 
the literature under other guises, but fortunately our research librarian enjoys these hard cases.) As before, each student must schedule an office hour before their library research talk and they receive peer feedback at this third talk.

Three weeks before finals the students submit their penultimate draft paper (10-20 pages plus appendices such as Maple output) which includes their own investigations and the library research. This draft must include at least one formal definition and theorem and proof (even when the theorems are trivial, the students can learn to write a clear proof). We also begin the final round of 45 minute talks; often a faculty member or a roommate or a parent will come to support the speaker. The final two students present during the scheduled final exam time slot, and all the students submit their final paper that same day.

My colleagues have offered the seminar in a variety of other formats. Some begin with a week or two of professorial presentations, followed by several weeks of student presentations on selections from a text, ending with three to five week student group projects. A few have tried Moorelike methods (with varying success), and a couple have tried problem solving seminars. Past seminar topics include: Dynamical Systems, Data analysis, Hilbert Spaces, Cartography, Mathematical Modeling, Cryptography, Monte-Carlo Methods in Financial Mathematics, Explorations Using Maple, Statistics and Sports, and Markov Chains.

Our advice: Applied topics tend to work better than theoretical ones. About half of my colleagues have students work in teams, while the other half use individual projects (students seem to prefer to work individually). Other advice: the first couple years of the seminar we tried to steer our better students towards big topics such as the Riemann hypothesis or Dirichlet's proof of an infinitude of primes in an arithmetic progression, but we found it is better to have students pick a topic that motivates them. Most of us allow a student with a special interest to pursue that regardless of the seminar course subtitle; for instance, a mathematics education major wrote an outstanding paper describing theoretical constructs for categories of middle school word problems, while a double major in math and biology studied the fractal dimension of blood vessel 
growth in cancerous tumors.

But our main advice we learned the hard way: have multiple talks, multiple paper submissions, and multiple required office hours, not just a midterm and final talk and paper. Checkpoints every couple weeks (a paper draft, talk, or an office hour) seem sufficient to maintain student momentum. When student projects go bad, student disengagement causes the failure, not the particular problem the student chose.

We highly recommend both oral and written presentations. Although Villanova University attracts excellent students, most of them go into teaching or business, not a graduate program in mathematics. During the senior exit interviews, the students sometimes comment on the value of the oral presentations to prepare them for the business world. The oral presentations also stimulate better quality work from the students; the judgment of their peers motivates students more than the judgment of a professor.

Examples of class handouts appear on the author's website [4].

Acknowledgement: The author is grateful to the referees for many suggestions that substantially improved the article.

\section{REFERENCES}

[1] Richard Guy. Unsolved Problems in Number Theory. Third edition. New York: Springer Verlag. 2004.

[2] Daniel Lyons. Smallest Numbers Beginning Sequences of 14 and 15 Consecutive Happy Numbers, accepted by Involve: a journal of mathematics. Accessed 30 Oct 2012.

[3] Stephanie Perez, Robert Styer. "Persistence: a Digit Problem." submitted to Involve: a journal of mathematics.

[4] http://www.homepage.villanova. edu/robert.styer/SeniorSeminar/seminar.html 


\section{BIOGRAPHICAL SKETCH}

Robert Styer teaches mathematics at Villanova University and also directs the Comprehensive Science program. He was a student of H. M. Stark who taught him to love and respect computational number theory, and Styer hopes he is passing that same love on to his own students. In his spare time, Styer helps his wife raise their six children. 